\title{
A single-center analysis of the survival benefits of adjuvant gemcitabine chemotherapy for biliary tract cancer.
}

\section{$\operatorname{AUTHOR}(S)$ :}

Yamanaka, Kenya; Hatano, Etsuro; Kanai, Masashi; Tanaka, Shiro; Yamamoto, Keiichi; Narita, Masato; Nagata, Hiromitsu; ... Machimoto, Takahumi; Taura, Kojiro; Uemoto, Shinji

\section{CITATION:}

Yamanaka, Kenya ... [et al]. A single-center analysis of the survival benefits of adjuvant gemcitabine chemotherapy for biliary tract cancer.. International journal of clinical oncology 2014, 19(3): 485-489

\section{ISSUE DATE:}

2014-06

URL:

http://hdl.handle.net/2433/199895

\section{RIGHT:}

The final publication is available at Springer via http://dx.doi.org/10.1007/s10147-0130578-X.; この論文は出版社版でありません。引用の際には出版社版をご確認ご利用くだ さい。; This is not the published version. Please cite only the published version. 


\section{A Single-center Analysis of the Survival Benefits of Adjuvant Gemcitabine}

\section{Chemotherapy for Biliary Tract Cancer}

Kenya Yamanaka ${ }^{1}$, Etsuro Hatano ${ }^{1}$, Masashi Kanai², Shiro Tanaka ${ }^{3}$, Keiichi Yamamoto ${ }^{3}$, Masato

Narita $^{1}$, Hiromitsu Nagata ${ }^{1}$, Takamichi Ishii ${ }^{1}$, Takahumi Machimoto ${ }^{1}$, Kojiro Taura ${ }^{1}$, Shinji Uemoto ${ }^{1}$

${ }^{1}$ Department of Surgery, Graduate School of Medicine, Kyoto University, Kyoto, Japan

${ }^{2}$ Outpatient Oncology Unit, Kyoto University Hospital, Kyoto, Japan

${ }^{3}$ Department of Clinical Trial Design and Management, Translational Research Center, Kyoto

University Hospital, Kyoto, Japan

\section{Address correspondence to:}

Etsuro Hatano, MD, $\mathrm{PhD}$

Department of Surgery, Graduate School of Medicine, Kyoto University

54 Kawaharacho, Shogoin, Sakyo-ku, Kyoto 606-8507, Japan

E-mail: etsu@kuhp.kyoto-u.ac.jp, Tel: 81-75-751-4323, Fax: 81-75-751-4348 


\section{Abstract}

Background Surgical resection is the only curative treatment of biliary tract cancer. However, the prognosis of biliary tract cancer is unsatisfactory. The aim of this study was to evaluate the benefits of adjuvant gemcitabine chemotherapy for biliary tract cancer.

Methods We performed a historical cohort study that involved 198 patients who underwent R0 surgical resections._Patients who underwent major hepatectomies were administered biweekly intravenous gemcitabine at a dose of $800 \mathrm{mg} / \mathrm{m} 2$. Otherwise, patients were administered intravenous gemcitabine at a dose of $1,000 \mathrm{mg} / \mathrm{m} 2$ in 3 weekly infusions, which were followed by a 1-week pause. The primary outcome was overall survival. The hazard ratio of adjuvant chemotherapy was estimated by propensity score-stratified Cox regression that was adjusted for confounders.

Results Forty patients received adjuvant chemotherapy. The hazard ratio of adjuvant chemotherapy was 0.47 (95\% confidence interval, $0.28-0.95 ; \mathrm{P}=0.03$ ). The subgroup analysis showed that the survival benefits were possibly modified by lymph node positivity (hazard ratio , 0.19; 95\% confidence interval, 0.07-0.58; interaction, $\mathrm{P}=0.22$ ), Stage III (hazard ratio, 0.11; 95\% confidence interval, 0.02-0.50; interaction, $\mathrm{P}<0.01$ ), intrahepatic cholangiocarcinoma (hazard ratio, 0.09; 95\% confidence interval , 0.01-0.67; interaction, $\mathrm{P}=0.05$ ), and a poorly differentiated tumor (hazard ratio , 0.16; 95\% confidence interval, 0.03-0.85; interaction, $\mathrm{P}=0.13$ ).

Conclusions Adjuvant gemcitabine chemotherapy for biliary tract cancer may be effective, 
particularly for patients with Stage III and intrahepatic cholangiocarcinoma.

\section{Mini-abstract}

Adjuvant gemcitabine chemotherapy for biliary tract cancer may be effective, particularly for patients with Stage III and intrahepatic cholangiocarcinoma. Biweekly $800 \mathrm{mg} / \mathrm{m} 2$ gemcitabine to patients with major hepatectomies was tolerable.

Key words: gemcitabine, adjuvant chemotherapy, biliary tract cancer

2017 Words, 1 Table, 2 Figures 


\section{Introduction}

Biliary tract cancer (BTC) is a common cancer, and approximately 15,000 people have died of BTC in Japan in a year., ${ }^{1,2}$ BTC was recently thought to be composed of intrahepatic cholangiocarcinoma (ICC), extrahepatic cholangiocarcinoma (ECC), gallbladder cancer, and ampulla of Vater cancer. Surgical resection is the only curative treatment of BTC. However, $60 \%$ of the patients with hilar cholangiocarcinomas developed distant metastasis after R0 resection. ${ }^{3}$ The 5-year survival rate for resectable patients is around $30 \% \cdot{ }^{4-6}$ The prognosis of BTC is unsatisfactory, and adjuvant chemotherapy is expected to improve the long-term survival of these patients.

Several chemotherapeutic regimens have been tested for unresectable BTC.

Chemotherapeutic agents, such as gemcitabine (GEM), 5-fluorouracil (5-FU), oxaliplatin, and cisplatin (CDDP) have survival benefits for patients with advanced BTC. ${ }^{7-10}$ Among them, GEM is the most promising agent for BTC. We previously conducted a phase-I study in order to determine the maximum-tolerated dose and the recommended dose of GEM for resected BTC (UMIN000004682). The results of the study indicated that patients who underwent surgical resections for BTC could not tolerate the standard dose and schedule of GEM for unresectable BTC. In particular, patients who underwent major hepatectomies hardly received an administration on day 8 because of neutropenia.

Thus, we decided to administer biweekly intravenous GEM at a dose of $800 \mathrm{mg} / \mathrm{m} 2$ to 
patients who underwent major hepatectomies in clinical settings. The aim of this study was to

evaluate the survival benefits of adjuvant GEM chemotherapy for BTC.

\section{Patients and methods}

\section{Study design and eligibility criteria}

The Kyoto University Graduate School and Faculty of Medicine Ethics Committee approved this study (E-1229) in accordance with the ethics guidelines for epidemiologic studies in Japan. We performed a historical cohort study in order to assess the survival benefits of adjuvant GEM chemotherapy for BTC. Patients at Kyoto University Hospital who were diagnosed with BTC and who underwent surgical resections between June 2001 and August 2010 were selected for this study. Among the 273 consecutive patients who underwent surgical resections for BTC, 211 patients had R0 surgical resections. The following 10 patients were excluded because they were treated with the following other chemotherapeutic agents in an adjuvant setting: 3 patients, Tegafur-uracil; 5 patients, leucovorin/5-FU; and 2 patients, 5-FU/CDDP. Three patients who died in hospital after the surgical resections were also excluded. Thus, 198 patients were included in this study. We basically performed adjuvant GEM chemotherapy except for patients with Stage-I- BCT, because even the prognosis for the patients with stage-II-BCT was unsatisfactory. 


\section{Adjuvant Chemotherapeutic Regimen}

The adjuvant GEM chemotherapy was started within 12 weeks after the surgical resection.

Before the administration of GEM, we confirmed that all drainage tubes were removed and that no abscesses were observed in the abdominal cavity. Patients who underwent major hepatectomies were treated with biweekly intravenous GEM at a dose of $800 \mathrm{mg} / \mathrm{m} 2$. Patients who did not undergo hepatectomies were treated with intravenous GEM at a dose of 1,000 mg/m2 in 3 weekly infusions, which were followed by a 1-week pause. The dose and schedule of chemotherapy was adjusted by individual physicians in response to the adverse events that were observed in the previous cycle. Toxicity was assessed based on the National Cancer Institute Common Toxicity Criteria scale, version 4.

\section{Statistical analysis}

The primary outcome measure was overall survival (OS), which was defined as the time from the date of surgery to the date of death from any cause. All patients were followed until August 2011. Patients who failed to undergo follow-up procedures were censored on the last day when they were confirmed to be alive.

In the primary analysis, bias due to confounding factors was adjusted for propensity score stratification, which stratifies patients according to their propensity scores, the probability that a 
patient would undergo adjuvant GEM chemotherapy. This method attempts to mimic randomization by creating strata of patients that received the adjuvant chemotherapy that is comparable on all observed confounders to strata of patients that did not receive the adjuvant chemotherapy. We estimated the propensity score for each patient by a multivariable logistic regression that included age, gender, Child-Pugh classification, primary lesion, $\mathrm{T}$ factor, $\mathrm{N}$ factor, Stage, tumor differentiation, operative procedures, and postoperative complications as confounders. These variables, other than the Child-Pugh classification, were examined after the surgical resection. The T factor, $\mathrm{N}$ factor, and Stage were estimated based on the International Union Against Cancer classification, 7th edition. The $\mathrm{C}$ statistics of the multivariate logistic regression was 0.760 . The hazard ratios (HR) for the OS of the adjuvant chemotherapy group compared to those for the OS of the nonadjuvant chemotherapy group were estimated by Cox proportional hazard models that were stratified to the quintiles of the propensity scores. We also performed a subgroup analysis that was stratified for the clinical variables by using a test of the interaction terms in the Cox proportional hazard models, unless a stratum included less than 5 patients in either group. We used chi-square tests (without Yates' correction) for the categorical comparisons of patient characteristics. The probability of survival was calculated by the Kaplan-Meier method. A P-value less than 0.05 indicated statistical significance. All tests were two-tailed. JMP for Windows software ver. 8.0 and SAS ver.9.2 were used for all statistical analyses. 


\section{Results}

\section{Patients}

Forty patients received adjuvant GEM chemotherapy (the adjuvant chemotherapy group)

and 158 patients did not receive any adjuvant chemotherapy (the nonadjuvant chemotherapy group).

There were significant differences in age, $\mathrm{T}$ factor, and Stage between the groups. Patients in the adjuvant chemotherapy group were younger and had higher Stage of BTC. No significant differences were present in the primary lesion, operative procedure, or postoperative complications. The patient characteristics are shown in Table 1.

\section{Recurrence}

The probability of recurrence was $41.8 \%$ (66/158) in the nonadjuvant chemotherapy group and 57.5\% (23/40) in the adjuvant chemotherapy group. Twenty-eight patients in the nonadjuvant chemotherapy group were administered GEM after tumor recurrence. The probabilities of the 1-, 2and 3-year survival of the patients with recurrent BTC who were administered GEM chemotherapy in the nonadjuvant chemotherapy group were $88.9 \%, 69.8 \%$, and $46.8 \%$, respectively. Patients in the adjuvant chemotherapy tended to have a longer life expectancy without recurrence, although this was not significant $(\mathrm{P}=0.16)$.

\section{Survival}


During the follow-up period, which had a median length of 3.2 years, 13 patients in the adjuvant chemotherapy group and 66 patients in the nonadjuvant chemotherapy group died. The probabilities of 1-, 2-, and 3-year survival in the adjuvant chemotherapy group were 97.5\%, 79.6\%, and $68.0 \%$, while those in the nonadjuvant chemotherapy group were $87.1 \%, 76.9 \%$, and $68.7 \%$, respectively (Figure 1). These survival probabilities were not comparable as the adjuvant chemotherapy group included patients with higher stages. In the propensity score analysis that was adjusted for the differential patient characteristics, the HR was 0.47 (95\% confidence interval [CI], 0.23-0.95; $\mathrm{P}=0.04$ ), suggesting a significant increase in survival by the administration of adjuvant GEM chemotherapy. The subgroup analysis according to the clinical variables is presented in Figure 2. The HRs were lower in female patients (HR, 0.17; 95\%CI, 0.05-0.60; interaction, $\mathrm{P}=0.90$ ), patients with ICC (HR, 0.09; 95\% CI, 0.01-0.67; interaction, $\mathrm{P}=0.05$ ), patients with lymph node positivity (HR, 0.19; 95\% CI, 0.07-0.58; interaction, $\mathrm{P}=0.22$ ), patients with Stage III BTC (HR, 0.11; 95\% CI, 0.02-0.50; interaction, $\mathrm{P}<0.01$ ), and patients with poorly differentiated tumors (HR, 0.16 ; $95 \%$ CI, $0.03-0.85$; interaction, $\mathrm{P}=0.13$ ). In the patients with ICC, the probability of 2-year survival in the adjuvant chemotherapy group was $91.7 \%$, while that in the nonadjuvant chemotherapy group was $68.2 \%(\mathrm{P}=0.04)$.

\section{Adverse events}

The grade of toxicity was not estimated for 4 patients because they were not administered 
adjuvant GEM chemotherapy at Kyoto University. Toxicities of grade 3 or more were observed in 13 patients (13/36, 36.1\%); neutropenia in 8 patients, thrombocytopenia in 2 patients, and other events (liver abscess, pancreatitis, vertigo) in 1 patient each. The toxicities were seen in 10 patients with hepatectomy (10/28, 35.7\%) and 3 patients without hepatectomy (3/8, 37.5\%), respectively. Two patients (2/36, 5.6\%) had grade-4 hematological toxicities. There were no treatment-related deaths.

\section{Discussion}

A GEM-based regimen is the main chemotherapeutic agent for BTC. GEM combined with CDDP has recently been used as the first-line treatment for unresectable BTC in clinical practice due to the results of the ABC-02 trial. ${ }^{8}$ There have been few articles regarding adjuvant chemotherapy for BTC. Adjuvant GEM plus S-1 chemotherapy has been reported to improve outcomes after surgical resections of BTC. ${ }^{11}$ A phase-III trial indicated that adjuvant mitomycin $\mathrm{C}$ and 5-FU chemotherapy may have survival benefits in patients with gallbladder cancer who underwent noncurative resections. ${ }^{12}$ However, prospective trials have not been reported concerning adjuvant GEM chemotherapy. Therefore, there is no definitive regimen of adjuvant chemotherapy for BTC and the efficacy of the adjuvant chemotherapy has not been established. However, there have been several reports of adjuvant radiation and chemoradiation therapy for BTC, and some of them may be promising. ${ }^{13-15}$ 
In cases of adjuvant GEM chemotherapy in patients who underwent resections of pancreatic cancer, $62 \%$ of the patients were given the full number of cycles of intravenous GEM, which consisted of a dose of $1,000 \mathrm{mg} / \mathrm{m} 2$ in 3 weekly infusions, which was followed by a 1-week pause, for 6 cycles. ${ }^{16}$ The average weekly dose was $700 \mathrm{mg} / \mathrm{m} 2$, and the median relative-dose intensity was $86 \% .{ }^{16}$ However, the phase-I study we previously conducted revealed that patients who were resected for BTC could not tolerate the standard dose. GEM is catalyzed to inactivate metabolite dFdU by cytidine deaminas in the liver. Patients with elevated bilirubin levels had significant deterioration in liver function after GEM therapy and a dose reduction is recommended. ${ }^{17}$ We have clinically administered biweekly intravenous GEM at a dose of $800 \mathrm{mg} / \mathrm{m} 2$ to patients who underwent major hepatectomies. This dose reduction was well tolerated. The HR of patients with hepatectomies was 0.60 (95\% CI, 0.28-1.29) in this study. Therefore, we think that this dose reduction regimen is useful for patients who underwent major hepatectomies for BTC.

A randomized phase-III trial of adjuvant chemotherapy with GEM vs. observations in patients with resectable bile duct cancer (UMIN000000820) is now being conducted in Japan. This study is examining whether adjuvant GEM chemotherapy increases the life expectancy of patients with bile duct cancer. However, ICC is not included in the eligibility criteria of this study. In patients with ICC who underwent surgical resections, it has been reported that an aggressive approach to recurrence can significantly prolong survival. ${ }^{18}$ However, there is no effective adjuvant regimen for 
ICC. ${ }^{19}$ This study indicated that adjuvant GEM chemotherapy was significantly associated with an increased OS of patients with ICC. It is highly expected that adjuvant GEM chemotherapy may have survival benefits for patients with ICC. Furthermore, previous studies have revealed that lymph node metastasis is an important prognostic factor in BTC. ${ }^{4,6}$ Patients with lymph node metastasis are required to be treated with adjuvant therapies. The subgroup analysis showed that adjuvant GEM chemotherapy improved the OS of patients with lymph node positivity and patients with Stage III. Further assessments of the effects of adjuvant GEM chemotherapy are needed to overcome the poor prognosis of patients with lymph node metastasis.

There were some limitations to this study. This study was a retrospective study, and the effects of adjuvant chemotherapy could not be directly evaluated, although the associations could be evaluated. We included postoperative complications and age in the clinical variables because they are thought to be related to the selection of adjuvant chemotherapy. The HR of adjuvant chemotherapy was estimated by a propensity score-stratified Cox regression, which was adjusted for confounders. However, unselected variables are not adjusted that might affect the survival benefits of adjuvant GEM chemotherapy.

In conclusion, adjuvant GEM chemotherapy may be effective for patients with BCT who underwent R0 resection, particularly for patients with Stage III and ICC. Biweekly intravenous GEM at a dose of $800 \mathrm{mg} / \mathrm{m} 2$ to patients who underwent major hepatectomies was tolerable. A prospective 
clinical trial of adjuvant GEM chemotherapy of BTC is needed to confirm these results, and it

should be designed to include lymph node and primary lesions in addition to ICC as prescribed

baseline factors.

\section{Conflict of interest}

The authors declare that they have no conflict of interest. 


\section{References}

1. de Groen PC, Gores GJ, LaRusso NF, et al. (1999) Biliary tract cancers. N Engl J Med

341:1368-1378

2. Okusaka T. (2002) Chemotherapy for biliary tract cancer in Japan. Semin Oncol 29:51-53

3. Hasegawa S, Ikai I, Fujii H, et al. (2007) Surgical resection of hilar cholangiocarcinoma: analysis of survival and postoperative complications. World J Surg 31:1256-1263

4. Kondo S, Takada T, Miyazaki M, et al. (2008) Guidelines for the management of biliary tract and ampullary carcinomas: surgical treatment. J Hepatobiliary Pancreat Surg 15:41-54

5. Nagino M, Kamiya J, Nishio H, et al. (2006) Two hundred forty consecutive portal vein embolizations before extended hepatectomy for biliary cancer: surgical outcome and long-term follow-up. Ann Surg 243:364-372

6. Kitamura K, Hatano E, Higashi T, et al. (2011) Prognostic value of (18)F-fluorodeoxyglucose positron emission tomography in patients with extrahepatic bile duct cancer. J Hepatobiliary Pancreat Sci 18:39-46

7. Kanai M, Yoshimura K, Tsumura T, et al. (2011) A multi-institution phase II study of gemcitabine/S-1 combination chemotherapy for patients with advanced biliary tract cancer. Cancer Chemother Pharmacol 67:1429-1434

8. Valle J, Wasan H, Palmer DH, et al. (2010) Cisplatin plus gemcitabine versus gemcitabine for 
biliary tract cancer. N Engl J Med 362:1273-1281

9. Yonemoto N, Furuse J, Okusaka T, et al. (2007) A multi-center retrospective analysis of survival benefits of chemotherapy for unresectable biliary tract cancer. Jpn J Clin Oncol 37:843-851

10. Verderame F, Russo A, Di Leo R, et al. (2006) Gemcitabine and oxaliplatin combination chemotherapy in advanced biliary tract cancers. Ann Oncol 17 :68-72

11. Murakami Y, Uemura K, Sudo T, et al. (2009) Adjuvant gemcitabine plus S-1 chemotherapy improves survival after aggressive surgical resection for advanced biliary carcinoma. Ann Surg 250:950-6

12. Takada T, Amano H, Yasuda H, et al. (2002) Is postoperative adjuvant chemotherapy useful for gallbladder carcinoma? A phase III multicenter prospective randomized controlled trial in patients with resected pancreaticobiliary carcinoma. Cancer 95:1685-1695

13. Gold DG, Miller RC, Haddock MG, et al. (2009) Adjuvant therapy for gallbladder carcinoma: the Mayo Clinic Experience. Int J Radiat Oncol Biol Phys 75:150-155 14. Kim K, Chie EK, Jang JY, et al. (2009) Role of adjuvant chemoradiotherapy for ampulla of Vater cancer. Int J Radiat Oncol Biol Phys 75:436-441

15. Kim TH, Han SS, Park SJ, et al. (2011) Role of adjuvant chemoradiotherapy for resected extrahepatic biliary tract cancer. Int J Radiat Oncol Biol Phys 81:853-859.

16. Oettle H, Post S, Neuhaus P, et al. (2007) Adjuvant chemotherapy with gemcitabine vs 
observation in patients undergoing curative-intent resection of pancreatic cancer: a randomized

controlled trial. JAMA 297:267-277

17. Venook AP, Egorin MJ, Rosner GL, et al. (2000) Phase I and pharmacokinetic trial of

gemcitabine in patients with hepatic or renal dysfunction: Cancer and Leukemia Group B 9565. J

Clin Oncol 18:2780-2787

18. Ercolani G, Vetrone G, Grazi GL, et al. (2010) Intrahepatic cholangiocarcinoma: primary liver resection and aggressive multimodal treatment of recurrence significantly prolong survival. Ann

Surg 252:107-114

19. Yang J, Yan LN. (2008) Current status of intrahepatic cholangiocarcinoma. World J Gastroenterol

14:6289-6297 


\section{Figure legends}

Figure 1. Unadjusted overall survival of adjuvant gemcitabine chemotherapy compared to nonadjuvant chemotherapy

Figure 2. Hazard ratio of adjuvant gemcitabine chemotherapy compared to nonadjuvant chemotherapy according to the clinical variables 


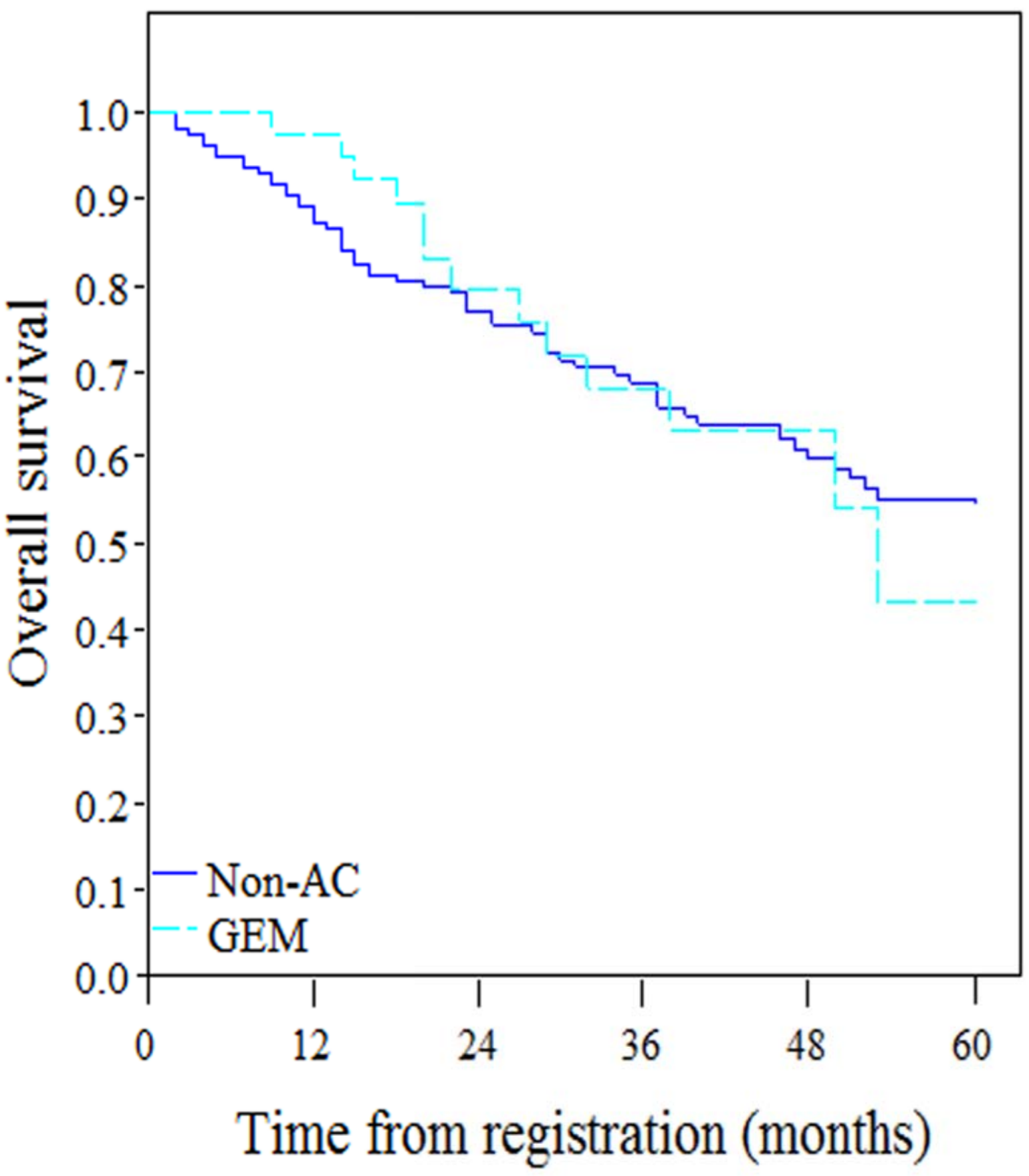

No. at Risk

Non-AC

GEM
158

137

40
103

24
75

15
55

8
42

1

Figure 1 


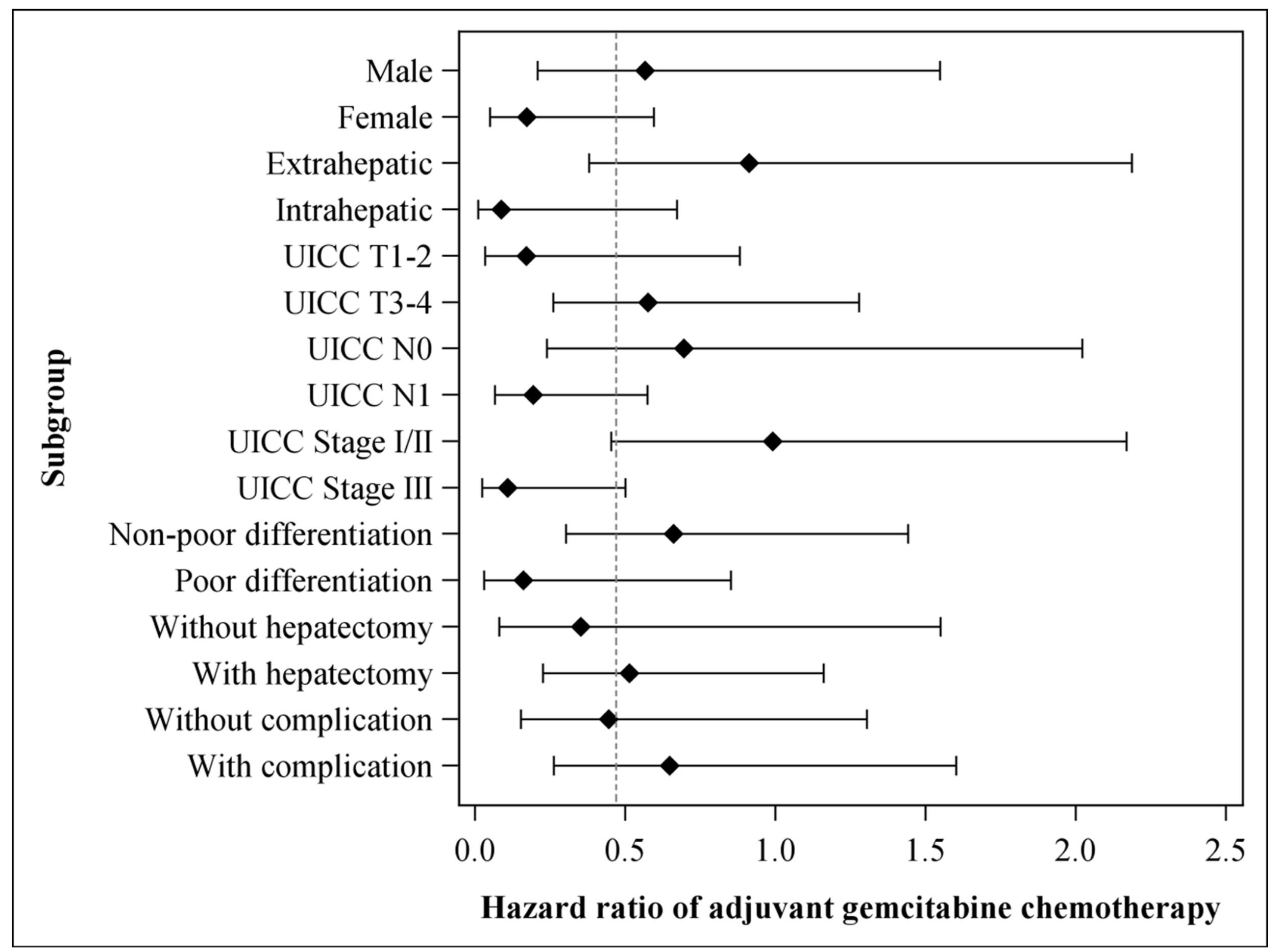

Figure 2 
Table 1. Patient characteristics

\begin{tabular}{|c|c|c|c|c|}
\hline & & $\begin{array}{l}\text { Nonadjuvant } \\
\text { chemotherapy } \\
(\mathrm{n}=158)\end{array}$ & $\begin{array}{l}\text { Adjuvant GEM } \\
\text { chemotherapy } \\
(\mathrm{n}=40)\end{array}$ & P-value \\
\hline \multirow[t]{2}{*}{ Age } & $<75$ & $121(76.6)$ & 39 (97.5) & $<0.01^{*}$ \\
\hline & $\geq 75$ & $37(23.4)$ & $1(2.5)$ & \\
\hline \multirow[t]{2}{*}{ Gender } & Male & $88(55.7)$ & $20(50.0)$ & 0.52 \\
\hline & Female & $70(44.3)$ & $20(50.0)$ & \\
\hline \multirow[t]{2}{*}{ Child-Pugh classification } & A & $134(84.8)$ & $36(90.0)$ & 0.39 \\
\hline & B & $24(15.2)$ & $4(10.0)$ & \\
\hline \multirow[t]{4}{*}{ Primary lesion } & Extrahepatic & $61(38.6)$ & $18(45.0)$ & 0.39 \\
\hline & Intrahepatic & $51(32.3)$ & $15(37.5)$ & \\
\hline & Gallbladder & $30(19.0)$ & $6(15.0)$ & \\
\hline & Ampulla of Vater & $16(10.1)$ & $1(2.5)$ & \\
\hline \multirow[t]{2}{*}{ UICC T } & T1-2 & $111(70.3)$ & $19(47.5)$ & $<0.01^{*}$ \\
\hline & T3-4 & $47(29.7)$ & $21(52.5)$ & \\
\hline \multirow[t]{2}{*}{ UICC N } & No & $120(76.0)$ & $25(62.5)$ & 0.09 \\
\hline & N1 & $38(24.0)$ & 15 (37.5) & \\
\hline \multirow[t]{3}{*}{ UICC Stage } & Stage I & $76(48.1)$ & $9(22.5)$ & $<0.01^{*}$ \\
\hline & Stage II & $60(38.0)$ & $19(47.5)$ & \\
\hline & Stage III & $22(13.9)$ & $12(30.0)$ & \\
\hline \multirow[t]{2}{*}{ Tumor differentiation } & Nonpoor & 138 (87.3) & $31(77.5)$ & 0.16 \\
\hline & Poor & $20(12.7)$ & $9(22.5)$ & \\
\hline \multirow[t]{2}{*}{ Operative procedures } & Without Hepatectomy & $47(29.8)$ & $10(25.0)$ & 0.55 \\
\hline & With Hepatectomy & $111(70.2)$ & $30(75.0)$ & \\
\hline \multirow[t]{2}{*}{ Postoperative complications } & Yes & $88(55.7)$ & $17(42.5)$ & 0.14 \\
\hline & No & $70(44.3)$ & $23(57.5)$ & \\
\hline
\end{tabular}

UICC, International Union Against Cancer; GEM, gemcitabine 